\title{
Magnetic fusion energy plasma diagnostic needs novel THz BWOs
}

\author{
Claudio Paoloni \\ Rosa Letizia \\ Mauro Mineo \\ Ali Malekabadi \\ Lancaster University \\ Lancaster United Kingdom \\ LA1 4YR
}

\author{
Neville C. Luhmann Jr \\ Branko Popovic \\ Logan Himes \\ Robert Barchfeld \\ Diana Gamzina \\ University of California Davis \\ Davis USA \\ Lingna Yue \\ Xiaopin Tang \\ University of Electronic Science \\ and Technology of China, \\ Chengdu, China
}

\author{
Jinjun Feng \\ Ye Tang, \\ Mengchao Gao \\ Fuzhi Zhang \\ Beijing Vacuum Electronic \\ Research Institute and Vacuum \\ Electronics National Lab, China
}

\begin{abstract}
The development of collective scattering diagnostics is essential for understanding of the anomalous transport attributed to short scale length microturbulence which poses a threat to the development of nuclear fusion reactors. Signals in the sub-THz range $(0.1-0.8 \mathrm{THz})$ with adequate power are required to probe the plasma. A joint international effort is therefore devoted to the design and realization of novel backward wave oscillators at $0.346 \mathrm{THz}$ and above with output power in the 1 Watt range to replace the bulky, high maintenance optically pumped FIR lasers so far utilized for this plasma diagnostic.
\end{abstract}

Keywords: plasma diagnostic, BWO, double staggered grating, double corrugated waveguide, terahertz.

\section{Introduction}

$\mathrm{THz}$ radiation is widely used in high $-k$ plasma collective scattering for plasma diagnostics in nuclear fusion reactors due to its properties to detect density fluctuation of electrons in the plasma and characterize the short scale length plasma turbulence [1]. The frequencies of interest, in case of the NSTX-U experiment at the Princeton Plasma Physics Laboratory, range from $0.346 \mathrm{THz}$ to $0.693 \mathrm{THz}$.

Presently, solid-state sources barely satisfy the power requirements or are limited in frequency $<0.3 \mathrm{THz}$. Bulky and expensive FIR lasers are the only available devices for the task. The need to extend the region of plasma to observe for a faster mapping requires new powerful and affordable THz sources. The potentiality of backward wave oscillators (BWOs) to provide relatively high power at $\mathrm{THz}$ frequencies has been demonstrated,

A joint international project is in progress to design and realize BWOs at $0.346 \mathrm{THz}$ operating frequency and above with output power of 100 's of $\mathrm{mW}$ to replace the optically pumped FIR lasers presently used in this plasma diagnostic. In the following, the approaches and the results so far obtained are discussed.

\section{The design}

A design strategy based on the test of different interaction structures to achieve performance with different fabrication approaches and cost has been adopted.

The design specifications are:

- $0.346 \mathrm{THz}$ operating frequency

- Output power of several hundreds of $\mathrm{mW}$

- Low cost

- Relatively easy assembly

- Compact volume (2 - $3 \mathrm{dm} 3)$

- Tunable (at least 5\%)

Slow wave structures: The double staggered grating [2] and the double corrugated waveguide [3] have been considered as slow wave structures. The folded waveguide was also evaluated, but the results were not satisfactory for its small value of backward wave coupling impedance [4].

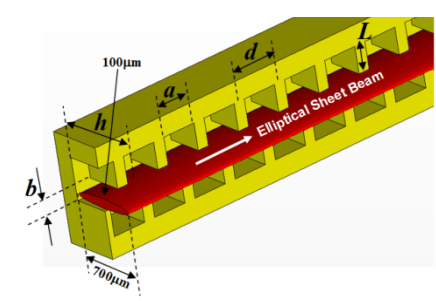

a)

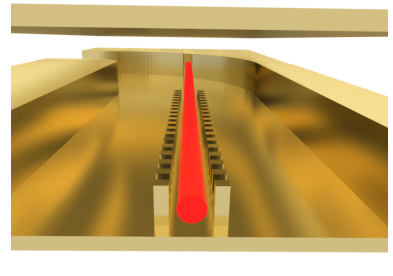

b)
Figure 1. a) double staggered grating; b) double corrugated waveguide 
The double staggered grating was designed to support an elliptical electron beam $300 \mu \mathrm{m}$ wide with a current of $16 \mathrm{~mA}$. The double corrugated waveguide was designed to support a cylindrical electron beam with $50 \mu \mathrm{m}$ radius and $10 \mathrm{~mA}$ current. Two BWOs were designed using the two interaction structures described above.

Three dimensional simulations by CST Particle Studio and MAGIC3D were performed. An average output power of $1 \mathrm{~W}$ was obtained with the double staggered grating, while in case of the double corrugated waveguide $0.45 \mathrm{~W}$ were obtained (Fig.2). As expected, the sheet beam approach provides higher power in comparison to a cylindrical beam approach. Depending on the output power specifications, both the SWSs are suitable for BWO fabrication. The SWSs are in the fabrication phase by the state of the art prototype NN1000 nano-CNC milling machine, developed by Digital Technology Laboratory (DTL), a subsidiary of DMG-Mori-Seki [5].
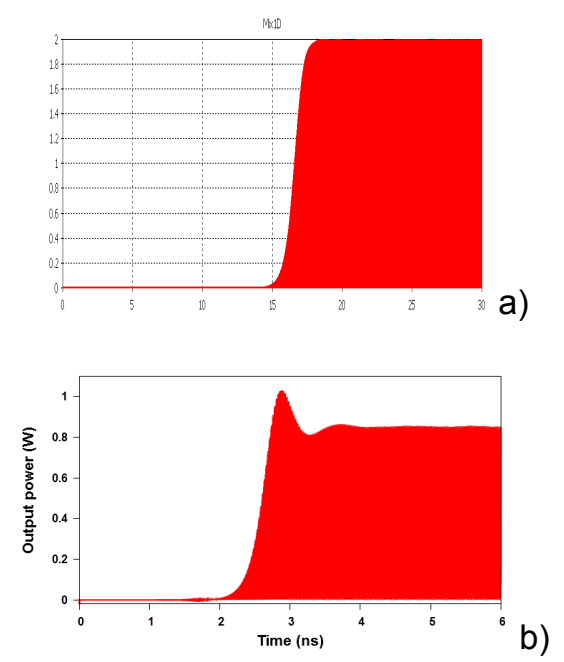

Figure 2. Output power: a) double staggered grating; b) double corrugated waveguide.

The electron gun: A planar cathode is considered to generate the cylindrical electron beam. A preliminary simulation and test was performed. A beam voltage of $11 \mathrm{kV}$ and a current of $45 \mathrm{~mA}$ have been obtained.

The elliptical electron beam will have a ratio 5.4:1 with a current density of $40 \mathrm{~A} / \mathrm{cm}^{2}$ and a $50 \%$ fill factor [6].

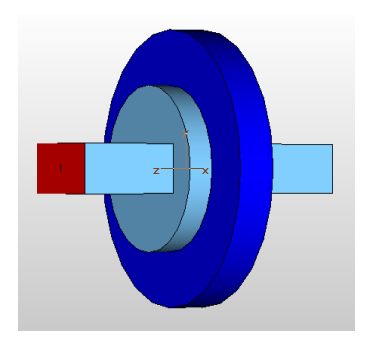

Figure 3. Window simulation setup
The magnetic focusing system: Different solutions based on solenoidal structures are under investigation to obtain up to $1.3 \mathrm{~T}$ for the full length of the BWOs.

The window: A window was designed using CST MWS (Fig.3) and tested in the frequency range $0.327-0.347$ THz. A VSWR below 1.2 was obtained. Further refinements are in progress.

\section{Conclusions}

The realization of novel $0.346 \mathrm{THz}$ BWOs is in progress. The performance at the state of the art will enable an improved plasma diagnostic for a deeper insight into the turbulence mechanism in nuclear fusion reactors.

\section{Acknowledgements}

This work is supported by EPSRC grant EP/L026597/1, US DOE Grants DE-FG02-99ER54518, DE-AC0209CH11466 and ATK's Alliance Partnership Program for MAGIC software.

\section{References}

1. Y. Ren, S.M. Kaye, E. Mazzucato, W. Guttenfelder, R.E. Bell, C.W. Domier, B.P. LeBlanc, K.C. Lee, N.C. Luhmann Jr., D.R. Smith, and H. Yuh, "Density Gradient Stabilization of Electron Temperature Gradient Driven Turbulence in a Spherical Tokamak," Physical Review Letters, vol. 106, 165005, April, 2011.

2. Y.-M. Shin, L. R. Barnett, and N. C. Luhmann, "Strongly confined plasmonic wave propagation through an ultrawideband staggered double grating waveguide," Applied Physics Letters, vol. 93, no. 22, p. 221504, 2008 .

3. M. Mineo and C. Paoloni, "Double-Corrugated Rectangular Waveguide Slow-Wave Structure for Terahertz Vacuum Devices," IEEE Transactions on Electron Devices, pp. 3169-3175, Nov. 2010.

4. Ye Tang, Mengchao Gao, Jinjun Feng, "The Analysis of Folded Waveguide and Double Corrugated Rectangular Waveguide for BWO”, 7th Europe/UKChina Millimeter Waves and Terahertz Technology Workshop, Chengdu, China, Sept. 2014

5. R. Barchfeld, D. Gamzina, A. Baig, L. R. Barnett, and N. C. Luhmann, "Nano CNC milling of two different designs of $0.22 \mathrm{THz}$ TWT circuits," in Vacuum Electronics Conference (IVEC), IEEE Thirteenth International, 2012, pp. 549-550.

6. Jinfeng Zhao, Na Li, Ji Li, Larry R. Barnett, Mike Banducci, Diana Gamzina, Zuhair A. Munir, and Neville C. Luhmann, Jr., "High Current Density and Long-Life Nanocomposite Scandate Dispenser Cathode Fabrication", IEEE Trans. on Electron Devices, April 2011. 